\title{
Molecular Dynamics Simulations of Energetic Ar Cluster Bombardment of $\mathrm{Ag}(111)$
}

\author{
G. PAlka ${ }^{a}$, L. RzezniK ${ }^{b}$, R. PARUCH ${ }^{a}$ AND Z. Postawa ${ }^{a, *}$ \\ ${ }^{a}$ Institute of Physics, Jagiellonian University, Kraków, Poland \\ ${ }^{b}$ University of Information Technology and Management, Rzeszów, Poland
}

\begin{abstract}
Large-scale molecular dynamics computer simulations are used to investigate the dynamics of material ejection during high-energy $\mathrm{Ar}_{n}$ cluster bombardment of $\mathrm{Ag}(111)$ at normal incidence. The silver sample containing 7 million atoms is bombarded with $\mathrm{Ar}_{n}$ projectiles $(n=45-30000)$ with kinetic energy spanning from a few keV up to $1 \mathrm{MeV}$. Such a wide range of projectile parameters allows probing processes taking place during low-density collision cascade as well as during high-density events characteristic of micrometeorite bombardment in space. The material modifications and total sputtering yield of ejected particles are investigated. While at low-energy impacts, ejection of individual silver atoms is the main emission channel, the ejection of large clusters from the corona of the created crater dominates for the high-energy impacts.
\end{abstract}

DOI: $10.12693 /$ APhysPolA.123.831

PACS: 68.49.Sf, 82.80.Ms, 83.10.Rs, 79.20.Rf

\section{Introduction}

Impacts of energetic projectiles on solid surfaces lead to material emission which is called sputtering. While processes that accompany impacts of atomic and small cluster projectiles on inorganic samples are well known, phenomena occurring during large cluster bombardment are still not fully understood [1,2]. While impact of atomic and small cluster projectiles like $\mathrm{Au}_{3}$ can be described by a concept of a linear collision cascade or thermal spike, processes taking place during impacts of larger clusters are more mesoscopic in nature. Consequently, they are better described by concepts adopted from hydrodynamics [3, 4].

Particularly interesting group of cluster projectiles are large gas clusters composed of Ar atoms. Due to unique properties of these projectiles, they have found numerous applications both in science and technology $[5,6]$. There are theoretical [7-12] and experimental studies [13-17] that investigate how the ejection efficiency of bombarded material depends on various parameters of cluster ion beam. In general, it has been observed that the total sputtering yield is an entangled function of the kinetic energy and size of the projectile. Mathematical form of this relation can be, however, greatly simplified if the total sputtering yield per projectile nucleon $\mathrm{Y}_{n}$ is plotted as a function of the kinetic energy per nucleon $\varepsilon$. In such representation, the data points corresponding to various kinetic energies and projectiles can be placed on a single curve $[7,8]$.

Most of the previous studies of Ar gas clusters impacts were limited to a relatively narrow range of kinetic

\footnotetext{
*corresponding author; e-mail: zbigniew.postawa@uj.edu.pl
}

energies and projectile sizes. In this study we investigate the effect of these parameters on the sputtering efficiency induced by an impact of $\mathrm{Ar}_{n}$ projectiles bombarding $\operatorname{Ag}(111)$ surface at normal incidence. The main goal of this study is to verify whether the relations observed previously will hold if a much wider range of the projectile parameters is used.

\section{Model}

Details of the MD computer simulations used to model cluster bombardment are described elsewhere [1]. The model of the silver crystal consists of approximately 7 million atoms arranged in a hemispherical sample of a radius $37 \mathrm{~nm}$. The $\mathrm{Ag}-\mathrm{Ag}$ interactions are described by the molecular dynamics/Monte Carlo corrected effective medium (MD/MC-CEM) potential for fcc metals [18]. The interactions between $\mathrm{Ar}$ atoms and $\mathrm{Ar}-\mathrm{Ag}$ atoms are described by the Lennard-Jones (L-J) potential splined with the $\mathrm{KrC}$ potential to properly model high-energy collisions. A wide range of $\mathrm{Ar}_{n}$ projectiles $(n=45-30000)$ is used to bombard $\operatorname{Ag}(111)$ surface at normal incidence. The projectiles have almost spherical shape with a diameter varying between approximately $1.4 \mathrm{~nm}$ and $12 \mathrm{~nm}$ for $\mathrm{Ar}_{45}$ and $\mathrm{Ar}_{30000}$, respectively. It is known that the motion induced by cluster projectile bombardment is mostly independent of the initial aiming point [1]. Consequently, only three trajectories were sampled for a given cluster size. The simulations are run at $0 \mathrm{~K}$ target temperature. The energy loss due to electronic excitations was introduced by using a dragging force that was proportional to velocity [19].

\section{Results and discussion}

The cross-sectional views of the temporal evolution of a typical collision event leading to ejection of silver atoms 
due to $1000 \mathrm{keV} \mathrm{Ar} 872$ bombardment are shown in Fig. 1. Several stages can be identified in the sputtering event. First, large energetic projectile is decelerated in the material leading to formation of a high density/high pressure region. Stress accumulated in this region is subsequently released leading to material relocation and development of pressure waves that propagate in the bombarded solid $[20,21]$. Formation of an almost hemispherical crater surrounded by a large corona composed of a liquidized metal is a consequence of this process (Fig. 1b). The process is mesoscopic in nature and can be described by a hydrodynamic flow model $[3,4]$. In this stage of sputtering material removal is dominated by azimuthally isotropic ejection of single atoms and small clusters from the crater. Later the crown forms protrusions that are several nanometers long. As shown in Fig. 1c large clusters are ejected from the ends of these protrusions. Finally, the crown shrinks to form the crater rim (Fig. 1d). The process of crater formation is similar for all investigated projectiles, with the exception of small projectiles $(n=45,60)$, where formation of vertically elongated pit is observed initially.
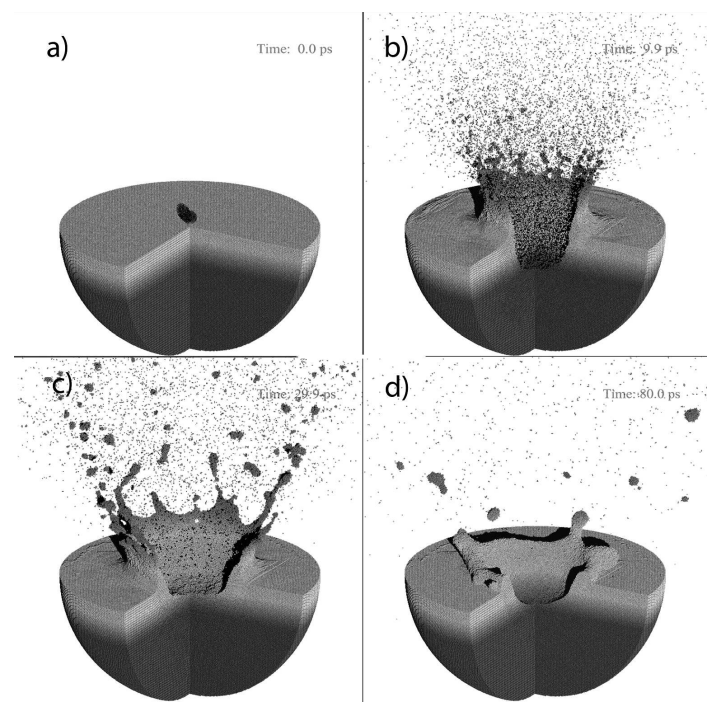

Fig. 1. Temporal evolution of the $\mathrm{Ag}(111)$ sample bombarded with $1000 \mathrm{keV} \mathrm{Ar}_{872}$ projectile at normal incidence. A frontal section of the sample was removed to illustrate energy deposition process.

As indicated in Fig. 1, an impact of energetic large projectile leads to significant material ejection. The calculated total sputtering yields induced by $\mathrm{Ar}_{n}$ bombardment at normal incidence are shown in Fig. 2. Indeed, ejection is massive. For instance, almost 50000 silver atoms are emitted by $1000 \mathrm{keV} \mathrm{Ar}_{872}$ projectiles. There are several trends that can be identified in the data. First, as shown in Fig. 2a, the total sputtering yield increases with the primary kinetic energy if the projectile size is kept constant. Such behaviour has already been reported in the previous studies with cluster projectiles $[7,8]$. It has been also observed that above certain thresh- old energy, the yield increases linearly with the kinetic energy $[7,8]$. Our results support this observation only partially. Indeed, the yield can be fitted with a straight line in a narrow energy range as shown in the inset to Fig. 2a. However, this quantity increases faster than linearly with the primary kinetic energy in a wider energy range.

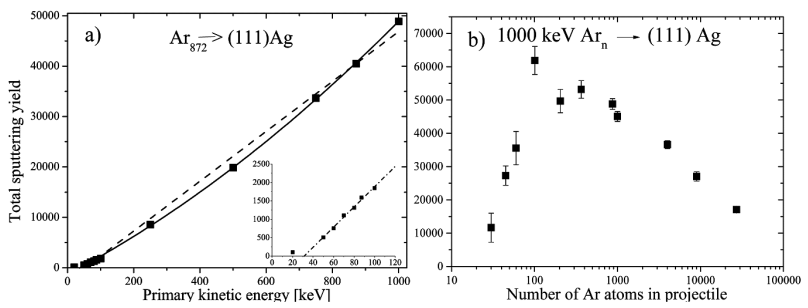

Fig. 2. Dependence of the total sputtering yield of silver atoms ejected from $\operatorname{Ag}(111)$ system on: (a) the primary kinetic energy of $\mathrm{Ar}_{872}$ projectile and (b) the cluster size of $1000 \mathrm{keV} \mathrm{Ar}_{n}$ projectiles. The inset in the part (a) shows the expanded region of small kinetic energy. Solid and dashed lines represent second and first power dependence on the primary kinetic energy. Dash-dotted line represents a linear fit to a low energy part of this dependence.

If the total kinetic energy of the projectile is constant, the yield first increases with the cluster size, reaches the maximum for $\mathrm{Ar}_{n}$ clusters containing a few hundred atoms and decreases for larger clusters as shown in Fig. 2b. Similar behaviour has been reported for $15 \mathrm{keV} \mathrm{C}_{n}$ projectiles bombarding the silver surface and explained by interplay between the range of the profile of deposited energy and the depth of the sample volume which contributes to sputtering (active volume) [22]. It has been shown that small clusters create a highly energized track but deposit most of their energy below the active volume. As the cluster size increases the yield gets larger because the energy deposition profile is shifted closer to the surface. In other words, more primary energy is deposited in the region from where particles can be emitted. However, if the projectile becomes too large, the lateral density of deposited energy decreases along with the yield. In the study presented in Ref. [22] it has been observed that the most efficient ejection occurs for $15 \mathrm{keV}$ $\mathrm{C}_{60}$ projectiles. However, it has been also observed that the size of the cluster stimulating the most efficient emission shifts towards larger clusters if the kinetic energy of the projectile is increased. It is not surprising, therefore, that in our study, where the kinetic energy of a projectile is $1000 \mathrm{keV}$, the most efficient ejection occurs for the clusters containing a few hundred atoms.

The data presented in Fig. 2 indicate that the erosion efficiency is an entangled function of both the cluster size and the total kinetic energy of a projectile. It has been observed, however, that the functional form of this dependence can be greatly simplified if the sputtering yield per single nucleon of the projectile $\mathrm{Y}_{n}$ is expressed as a function of projectile kinetic energy per nucleon of the 


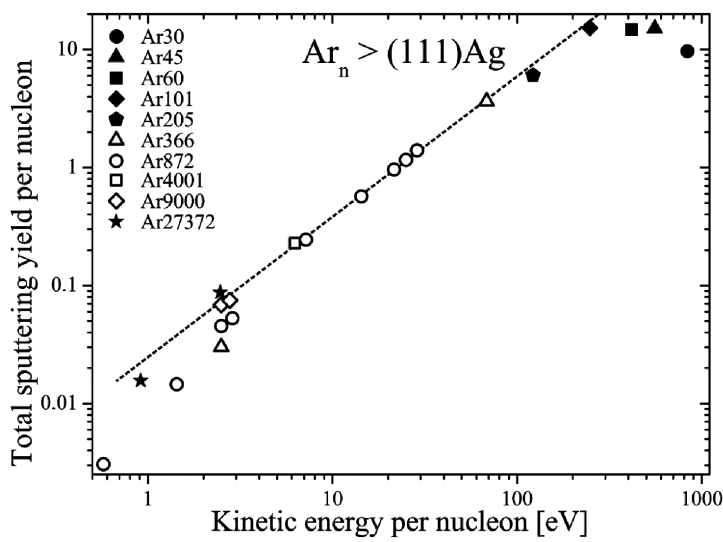

Fig. 3. Dependence of the total sputtering yield per projectile nucleon on the primary kinetic energy per nucleon for various Ar projectiles.

projectile $\varepsilon[7,8]$. In such representation, the data points for $\mathrm{Y}_{n}$ collected with various clusters and kinetic energies can be placed on a single curved line with a positive slope with $\varepsilon$. As shown in Fig. 3, our data support this observation again only partially. Indeed, below $\varepsilon \approx 300 \mathrm{eV} /$ nucleon $\mathrm{Y}_{n}$ dependence on $\varepsilon$ is similar to the trend reported in $[7,8]$. However, at larger $\varepsilon$ there is a distinctive deviation from this trend and ultimately the yield per nucleon even decreases with the increase of $\varepsilon$. It is visible in Fig. 3 that the deviation occurring at large $\varepsilon$ is caused by small cluster projectiles having high primary kinetic energy. As already discussed such clusters penetrate deep into the solid depositing part of their energy below the region from where silver atoms can be ejected. We observe, therefore, that the relation between $\mathrm{Y}_{n}$ and $\varepsilon$ is more complicated than assumed so far.

\section{Conclusions}

We have investigated the effect of the projectile size and its kinetic energy on the total sputtering yield of silver particles ejected from the $\mathrm{Ag}(111)$ surface bombarded by $\operatorname{Ar}_{n}$ projectiles at normal incidence. It has been observed that the relations between the sputtering efficiency and the projectile parameters may differ from their counterparts recorded in a narrower span of the primary energy. For instance the yield increases faster than linearly. Moreover, while there is still a single curve that describes the relation between $\mathrm{Y}_{n}$ and $\varepsilon$, its shape is more complicated than assumed so far.

\section{Acknowledgments}

The authors would like to thank the National Centre of Science program nos. 2011/01/D/ST4/05070, PB1839/ B/H03/2011/40 for their financial support of this research. The authors would also like to thank the PL-Grid
Infrastructure for the use of their computing resources and technical support.

\section{References}

[1] B.J. Garrison, Z. Postawa, Mass Spectrom. Rev. 27 289 (2008).

[2] R. Behrisch, K. Wittmaack, Top. Appl. Phys. 64, 1 (1991) and references therein.

[3] M.M. Jakas, E.M. Bringa, R.E. Johnson, Phys Rev. B 65, 165425 (2002).

[4] M.F. Russo, B.J. Garrison, Anal. Chem. 78, 7206 (2006).

[5] I. Yamada, J. Matsuo, N. Toyoda, Nucl. Instrum Methods Phys. Res. B-Beam Interact. Mater. Atoms 206, 820 (2003).

[6] A. Wucher, N. Winograd, Anal. Bioanal. Chem. 396, 105 (2010).

[7] C. Anders, H.M. Urbassek, R.E. Johnson, Phys. Rev. B 70, 155404 (2004).

[8] A. Delcorte, B.J. Garrison, K. Hamraoui, Anal. Chem. 81, 6676 (2009).

[9] T. Aoki, T. Seki, J. Matsuo, Z. Insepov, I. Yamada, Nucl. Instrum. Methods Phys. Res. B-Beam Interact. Mater. Atoms 153, 264 (1999).

[10] T. Aoki, T. Seki, J. Matsuo, Nucl. Instrum. Methods Phys. Res. B-Beam Interact. Mater. Atoms 267, 2999 (2009)

[11] T. Aoki, J. Matsuo, Nucl. Instrum. Methods Phys. Res. B-Beam Interact. Mater. Atoms 242, 517 (2006).

[12] B.J. Garrison, K.E. Ryan, Anal. Chem. 80, 5302 (2008).

[13] K. Ichiki, S. Ninomiya, Y. Nakata, Y. Honda, T. Seki, T. Aoki, J. Matsuo, Appl. Surf. Sci. 255, 1148 (2008).

[14] S. Ninomiya, Y. Nakata, K. Ichiki, T. Seki, T. Aoki, J. Matsuo, Nucl. Instrum. Methods Phys. Res. B-Beam Interact. Mater. Atoms 256, 493 (2007).

[15] S. Ninomiya, Y. Nakata, Y. Honda, K. Ichiki, T. Seki, T. Aoki, J. Matsuo, Appl. Surf. Sci. 255, 1588 (2008).

[16] J.L.S. Lee, S. Ninomiya, J. Matsuo, I.S. Gilmore, M.P. Seah, A.G. Shard, Anal. Chem. 82, 98 (2010).

[17] S. Rabbani, A.M. Barber, J.S. Fletcher, N.P. Lockyer, J.C. Vickerman, Anal. Chem. 83, 3793 (2011).

[18] C.L. Kelchner, D.M. Halstead, L.S. Perkins, N.M. Wallace, A.E. Depristo, Surf. Sci. 310, 425 (1994).

[19] J. Lindhard, M. Scharff, Phys. Rev. 124, 128 (1961).

[20] J. Samela, K. Nordlund, Nucl. Instrum. Methods Phys. Res. B-Beam Interact. Mater. Atoms 267, 2980 (2009).

[21] Z. Postawa, B. Czerwinski, M. Szewczyk, E.J. Smiley, N. Winograd, B.J. Garrison, Anal. Chem. 75, 4402 (2003).

[22] B. Czerwiński, L. Rzeznik, K. Stachura, R. Paruch, B.J. Garrison, Z. Postawa, Vacuum 82, 1120 (2008). 